\title{
CREENCIAS SOBRE LA INCLUSIÓN EDUCATIVA Y PRÁCTICAS DOCENTES EN EL CONTEXTO DE APRENDIZAJE DE LA LECTOESCRITURA INICIAL
}

\author{
Beliefs about Educational Inclusion and Teaching Practices \\ in the Context of Early Literacy Learning
}

\section{Carla Leticia Paz-Delgado ${ }^{1}$}

cpaz@upnfm.edu.hn

\section{Elma Barahona ${ }^{1}$}

ebarahona@upnfm.edu.hn

1 Universidad Pedagógica Nacional Francisco Morazan, Honduras

Fecha de recepción: 4/03/2020 Fecha de aprobación: 26/04/2020

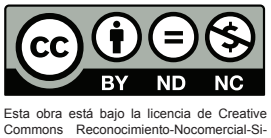

\section{Resumen}

La inclusión educativa es un enfoque que pretende eliminar las barreras que limitan el aprendizaje y la participación del alumnado, promoviendo la equidad y la justicia social. El objetivo de esta investigación fue analizar las creencias y prácticas pedagógicas de docentes hondureńos respecto a la inclusión educativa en el contexto de aprendizaje de la lectoescritura inicial (LEI). Para ello se diseñó una investigación con enfoque mixto. Se emplearon cuatro técnicas para recolectar datos: observaciones en aula, entrevistas estructuradas, un cuestionario para explorar creencias y prácticas pedagógicas de la LEI en el contexto de la inclusión educativa, y un taller investigativo. Se conformó una muestra de 25 docentes, 19 regulares y 6 de apoyo. Los resultados indican que el profesorado asume una perspectiva dilemática en las creencias sobre el potencial de aprendizaje de los estudiantes y una concepción interaccionista en el sistema de creencias sobre los apoyos educativos para el aprendizaje de la LEI en estudiantes de primer grado de educación básica. En cuanto a las prácticas inclusivas, el profesorado utiliza aquellas vinculadas con el manejo efectivo del aula y, en menor medida, las estrategias de agrupamiento, la enseñanza adicional, las adaptaciones curriculares y los apoyos para la participación. En conclusión, las creencias y prácticas del profesorado están centradas en la mirada del déficit de los estudiantes con diversidad en el aprendizaje. Estos hallazgos ponen en evidencia la creciente necesidad de promover de manera sistemática espacios de formación para docentes acerca de la inclusión educativa en el contexto de aprendizaje de la LEI a nivel nacional.

Palabras clave: creencias y prácticas docentes, inclusión educativa, justicia social, lectoescritura inicial.

\section{Abstract}

Educational inclusion is an approach that seeks to eliminate barriers that limit students from learning and participation, promoting equity and social justice. This research aims to analyze the beliefs and pedagogical practices of Honduran teachers, with respect to educational inclusion in the context of early grade literacy (EGL) learning. A mixed methods research approach was implemented. Four techniques were used to collect data: classroom observations, structured interviews, a questionnaire to explore beliefs and pedagogical practices of EGL in an educational inclusion context, and a research workshop. A sample of 25 teachers, 19 regular teachers and 6 support teachers was selected. The results indicate that teachers assume a dilemmatic perspective in beliefs about students' learning potential and an interactive perspective in their belief system about educational support for EGL learning in first graders. Regarding inclusive practices, teachers use those related to effective classroom management, and to a lesser extent, grouping strategies, additional instruction, curricular adaptations, and supports for participation. In conclusion, the beliefs and practices of the teachers are focused on the view of the deficit of the students. These findings highlight the growing need to systematically promote training opportunities for teachers in educational inclusion in the learning context of EGL at the national level.

Keywords: early grade literacy, inclusive education, social justice, teachers' beliefs and practices. 


\section{Introducción}

La concepción de la diversidad humana en educación ha evolucionado a lo largo de la historia, caracterizándose en un inicio por una respuesta institucionalizada que trajo consigo procesos de marginación y exclusión (Chiner \& Cardona, 2013). Debido a ello, una gran cantidad de docentes se ha formado con un paradigma homogeneizador, desconociendo que la diversidad es una característica inherente a la condición humana (Duk \& Murillo, 2011; García-Carrasco \& Canal-Bedia, 2018; Paz \& Cardona, 2018). En contraposición a esta mirada, el reconocimiento del valor de la diversidad sentó las bases para un cambio de enfoque que favorece la justicia social y la inclusión (Martínez-Pineda \& Soler-Martín, 2015; Montané, 2015). Esta perspectiva ofrece un marco favorable para garantizar la igualdad de oportunidades y la plena participación, pues contribuye a una educación más personalizada y al fomento de la colaboración entre todos los miembros de la comunidad educativa (Booth \& Ainscow, 2015; Durán \& Climent, 2017).

En el año 2014 se aprobó en Honduras el Reglamento de Educación Inclusiva para Personas con Discapacidad, Necesidades Educativas Especiales y Talentos Excepcionales (Secretaría de Educación, 2014), en el cual se establece la creación del programa Servicios Educativos de Atención a la Diversidad (SEAD). El programa está orientado a generar recursos y estrategias para disminuir y eliminar barreras de aprendizaje en los distintos ámbitos y áreas de conocimiento, para que la experiencia de aprendizaje sea justa, equitativa e incluyente. Actualmente se desconoce en qué medida este reglamento está siendo ejecutado por los centros educativos, además se desconocen los desafíos que enfrentan los docentes cuando aplican medidas que favorezcan la inclusión en el aula. Por tal razón, esta investigación analiza las creencias y prácticas de los docentes regulares y personal docente de apoyo respecto a la inclusión educativa en el contexto del aprendizaje de la lectoescritura inicial (LEI), habilidad fundacional que desempeńa un rol clave en el desarrollo integral de las personas. Así mismo, se examina el tipo de prácticas pedagógicas empleadas para favorecer el desarrollo de este proceso, desde la mirada de la inclusión y la justicia social.

Es importante destacar que, en Honduras, la investigación sobre los procesos de inclusión educativa es limitada, y que es aún más escasa la dedicada a identificar y analizar las creencias y prácticas del profesorado respecto al contexto de aprendizaje de la LEI. Por esta razón, se considera que esta investigación es inédita en el país y sienta precedentes para potenciar esta línea de investigación a nivel nacional y regional.

\section{Revisión de la literatura}

La revisión de la literatura incluye tres aspectos fundamentales que facilitan la comprensión de las categorías analizadas en esta investigación. El primer aspecto aborda la inclusión educativa y la justicia social, el segundo las creencias de los docentes sobre la inclusión educativa, y el tercero se enfoca en las prácticas pedagógicas para promover la inclusión educativa en el ámbito de la LEI.

\subsection{Inclusión educativa y justicia social}

La educación inclusiva se define como un proceso que permite responder a la diversidad de necesidades de los educandos a través de la identificación de las barreras de aprendizaje y participación (Booth \& Ainscow, 2015). Este enfoque trae consigo grandes desafíos y disyuntivas, pues implica la presencia, permanencia y participación de todo el alumnado en la vida escolar (Organización de las Naciones Unidas para la Educación, la Ciencias y la Cultura [UNESCO], 2017).

La evidencia científica derivada de la investigación educativa en este campo propone una variedad de elementos considerados indispensables en la construcción de espacios educativos inclusivos y que a su vez se caracterizan por su naturaleza dilemática, porque se trata de ofrecer una educación pertinente a las características individuales del alumnado en una estructura común, garantizando el respeto y aprecio por la diversidad (Duk \& Murillo, 2016; López et al., 2010; Norwich, 2019). Norwich (2014) clasifica los elementos dilemáticos en nueve categorías: 
a) el reconocimiento del valor de la diversidad humana;

b) la participación de todo el alumnado;

c) la reorganización escolar y la resolución de problemas;

d) la promoción de valores y culturas inclusivas;

e) la mejora de la igualdad de oportunidades;

f) la escucha de la voz del alumnado y su empoderamiento;

g) la inclusión como un proceso sin fin;

h) la educación inclusiva como medio para una sociedad inclusiva, $y$

i) las prácticas pedagógicas.

La consideración e integración de estos elementos en el contexto de la inclusión educativa favorece la implementación de un enfoque educativo que promueve el pleno desarrollo del alumnado, garantizando el derecho a la educación desde la perspectiva de la justicia social.

\subsection{Las creencias de los docentes sobre la inclusión educativa}

El estudio de las creencias de los docentes, relativas a la inclusión educativa, ha cobrado gran relevancia en los últimos años (López et al., 2009), debido al impacto de estas en las decisiones que involucran el proceso de enseñanza-aprendizaje y sus repercusiones en el desempeño del alumnado (Chiner et al., 2015). Se sabe que el sistema de creencias se establece desde antes de la formación inicial docente (Leko et al., 2015; Vaillant \& Marcelo, 2014), por tanto, su modificación solo será posible desde una preparación crítica y reflexiva, que permita el análisis de los antecedentes culturales y sociales así como de las experiencias previas y las características personales (Chiner et al., 2015).

Por tal razón, una gran parte de la investigación actual pone énfasis en el impacto de la formación inicial y permanente como factor determinante para la práctica en el aula (Olson \& Roberts 2018; Peebles
\& Mendaglio, 2014; Pegalajar \& Colmenero, 2017; Sharma, 2018; Simón et al., 2018) en el ámbito de la inclusión y la diversidad humana (Paz, 2017). Se resalta la necesidad de impulsar programas de formación que preparen al docente para atender a la diversidad de forma más efectiva (Chiner et al., 2015) por medio de la reflexión sobre las barreras de aprendizaje, y generar un clima positivo de aula (Florian \& Linklater 2010; Hamre \& Oyler, 2004; Pugach et al., 2020).

Muñoz et al. (2015) proponen que las creencias de los docentes sobre la inclusión educativa son de tres tipos: individuales, dilemáticas e interactivas. Bajo la perspectiva individual, se conciben las diferencias desde la mirada del déficit del estudiante, y la forma de respuesta recae exclusivamente en el profesor especialista. En la perspectiva dilemática, se reconoce que la educación es una cuestión de derechos humanos, pero al mismo tiempo se generan dilemas sobre la necesidad del diagnóstico, la organización escolar y la promoción de los aprendizajes. En la última perspectiva, la interactiva, la respuesta educativa es responsabilidad de toda la comunidad educativa y el trabajo colaborativo entre el personal docente se basa en la concepción de la diversidad como oportunidad educativa.

López et al. (2014) señalan que cuando un estudiante presenta una barrera de aprendizaje, por lo general se adjudica inmediatamente el déficit al propio educando, lo que deriva en una valoración de la diversidad como un problema y no como una oportunidad. Otros investigadores encontraron que cuando se asume la perspectiva interactiva, las creencias se orientan al reconocimiento y superación de las barreras para el aprendizaje, y se promueve la participación de los estudiantes (López et al., 2018).

Desde la perspectiva interactiva, las etiquetas que provienen del modelo clínico (médico) pasan a un segundo plano y se efectúa la actuación educativa desde modelos de alto nivel centrados en la construcción de un sistema escolar que potencie el aprendizaje de todos los estudiantes (el modelo de aprendizaje visible y el modelo de aprendizaje sin límite). Esta 
perspectiva, además, es compatible con la idea de la transformabilidad, propuesta por Hart et al. (2004), que hace referencia a un conjunto de firmes creencias sobre el potencial de cambio de las capacidades de aprendizaje del alumnado, generadas por las prácticas de enseñanza del profesorado (Figura 1).

\section{Figura 1. Perspectivas sobre la inclusión educativa}

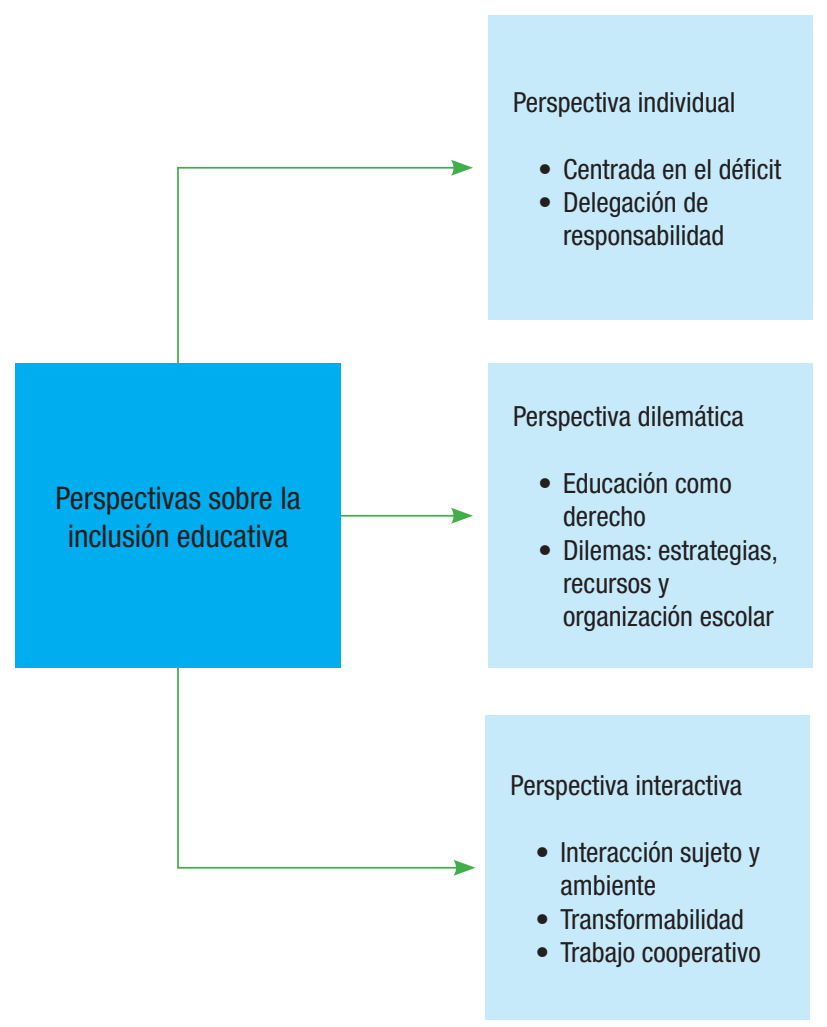

Nota: Adaptación de perspectivas sobre inclusión educativa de López et al. (2010)

\subsection{Prácticas pedagógicas inclusivas}

Las prácticas pedagógicas inclusivas que promueven la eliminación de las barreras de aprendizaje y participación se generan a partir de la cooperación y las altas expectativas, y propician el aprendizaje dialógico (Salvador, 2013). Un amplio grupo de estudios sobre la inclusión en educación se han orientado a determinar y a caracterizar aquellas prácticas efectivas en la atención del alumnado y su diversidad (Chiner \& Cardona, 2012; Flores-Barrera et al., 2017; Graham, 2016; McKay \& Manning, 2019).
Por su parte, Bravo (2010) afirma que las prácticas pedagógicas más efectivas se relacionan con el compromiso hacia el aprendizaje, el clima del aula, los apoyos a la participación y las conductas cooperativas y colaborativas. Además, seńala este autor que las estrategias efectivas son las de agrupamiento, las adaptaciones instructivas y del currículo de aprendizaje, el manejo efectivo del aula, la enseñanza adicional y los apoyos a la participación del alumnado en el proceso de aprendizaje (Cardona, 2003, 2006; Hoppey et al., 2018; Salvador, 2013).

De igual forma, el trabajo cooperativo entre el profesorado regular y el de apoyo constituye una práctica clave en el proceso de construir culturas y prácticas inclusivas (Muñoz et al., 2015). La investigación ha demostrado que los docentes que consideran tener suficiente apoyo desarrollan creencias y actitudes más favorables hacia la inclusión (Chiner \& Cardona, 2013).

A partir de la revisión de la literatura, nuestro estudio intenta responder las siguientes cuestiones de investigación: ¿cuáles son las creencias de los docentes sobre la inclusión educativa?; ¿`cuáles son las prácticas inclusivas que implementan los docentes en el aula de clase? y ¡cómo se configuran las creencias y las prácticas de los docentes para la inclusión educativa en el contexto específico de aprendizaje de la LEI?

\section{Método}

Se diseñó la investigación con un enfoque mixto con alcance exploratorio tomando como muestra un centro escolar. Este tipo de aproximación metodológica fue seleccionada para estudiar la naturaleza polifacética de las creencias y prácticas docentes, pues las mismas plantean enormes retos para su estudio (López et al., 2010). A partir del uso de una metodología participativa-colaborativa, se logró estudiar con mayor detalle la naturaleza de las creencias y prácticas de los docentes, lo que a su vez permitió reflexionar acerca del nivel de comprensión que estos tienen sobre el trabajo que desempeñan en un contexto específico de aprendizaje de la LEI. 


\subsection{Participantes}

El diseño de la investigación que incluye el proceso de selección y reclutamiento de los participantes de este estudio se sometió a la revisión del Comité de Ética de la Facultad de Educación de la Universidad del Valle de Guatemala, el cual dio su aval para la implementación del estudio. El proceso de reclutamiento incluyó el consentimiento informado para solicitar la participación voluntaria de los docentes. A su vez, se solicitó el consentimiento de las autoridades del centro y de los padres de familia, porque se requería acceder a las aulas de clase para el proceso de observación. Debido a la importancia de la triangulación de la información y como medio para garantizar la credibilidad de los hallazgos, se efectuaron dos etapas de selección de los participantes.

- Etapa I. En esta etapa se consideró la participación de todos los docentes del primer ciclo de educación básica (primero, segundo y tercer grado) del Centro de Investigación en Innovación Educativas (CIIE) creado para constituirse en el principal centro de práctica profesional de la Universidad Pedagógica Nacional Francisco Morazán (UPNFM) de Honduras. La selección de este grupo de docentes se efectuó para contar con una perspectiva general de las creencias y las prácticas de los docentes en el proceso de enseñanza de la LEI. En total, participaron 25 profesores.

- Etapa II. Para profundizar en temas claves identificados en la primera etapa del estudio, se trabajó con los 10 docentes responsables del primer grado. Los criterios de su inclusión fueron: a) nivel de responsabilidad en la dirección y gestión de procesos pedagógicos; b) al menos cuatro horas de docencia frente a estudiantes; y c) nivel de coordinación e implementación de procesos de atención a la diversidad en el aula de clases.

\subsection{Categorías de análisis}

El proceso de construcción de las categorías se realizó a priori, teniendo en consideración la literatura que sostiene este estudio. Se definieron dos categorías y las subcategorías correspondientes a cada una.
La primer categoría es creencias docentes y sus subcategorías son naturaleza de las diferencias humanas y respuesta educativa (López et al., 2009; López et al., 2014; Muñoz et al., 2015). La otra categoría es prácticas pedagógicas inclusivas y tiene cinco subcategorías: manejo efectivo del aula, estrategias de agrupamiento, enseñanza adicional, enseñanza adaptativa y apoyos a la participación (Cardona, 2003; Chiner \& Cardona 2012; Chiner et al., 2015).

\subsection{Instrumentos}

Se diseñó una batería de instrumentos tanto para el componente cualitativo como para el cuantitativo. Para el cualitativo se utilizó una ficha para el registro anecdótico de las observaciones de aula (permitió obtener información para analizar las prácticas docentes), un guion de trabajo para el taller investigativo (Quintana \& Montgomery, 2006) y una guía de preguntas semiestructuradas para la entrevista de docentes que brindan atención directa y diaria al alumnado del primer grado (ambos instrumentos están orientados al análisis de las creencias y prácticas docentes).

Con relación al componente cuantitativo, se construyó el cuestionario de creencias y prácticas pedagógicas inclusivas en contextos de aprendizaje de lectoescritura (diseñado por las autoras). Este cuestionario se elaboró a partir de dos instrumentos: el cuestionario de dilemas en los procesos de inclusión (López et al., 2010) y el cuestionario de adaptaciones instructivas (Cardona, 2003). Una vez elaborado el cuestionario, se procedió a su validación por siete jueces expertos en inclusión, con más de 10 años de experiencia en el campo. El indice de validez de contenido se obtuvo por medio del procedimiento de Lawshe. En el caso del cuestionario, el índice fue de 1.068, lo que indica un alto grado de validez en el contenido. Posteriormente se realizó un estudio piloto con 44 docentes de primer grado de escuelas públicas similares al CIIE, el coeficiente de alpha de Cronbach fue de .83 , lo que indica una buena coherencia interna de los ítems del cuestionario. 


\subsection{Procedimientos}

La investigación se desarrolló en las siguientes etapas.

3.3.1. Etapa 1. Se realizaron seis observaciones, con una duración de cuarenta minutos cada una. Fueron realizadas en diferentes momentos durante dos semanas, siguiendo el horario de clases, y se tomaron fotografías de las prácticas desarrolladas por los docentes observados.

3.3.2. Etapa 2. Se realizó un taller investigativo con una duración de 3 horas. Al inicio se aplicó el cuestionario. Seguidamente y haciendo uso de las fotografías de la Etapa 1 se generó un espacio de reflexión sobre las creencias y las prácticas mediante la técnica de investigación social y colaborativa "campos de fuerzas" (Chevalier, 2009).

3.3.3. Etapa 3. Para profundizar y triangular la información, se desarrollaron entrevistas con el personal docente involucrado directamente en el proceso de aprendizaje de la LEI en el primer grado del centro educativo.

\subsection{Procesamiento y análisis de datos}

Con relación a los datos cuantitativos, se emplearon pruebas descriptivas utilizando los programas SPSS y Excel. Para el análisis de datos cualitativos se empleó el ciclo de análisis de tres pasos: 1) reducción de los datos; 2) resumen estructurado para favorecer la reflexión; 3) elaboración y verificación de conclusiones. Para el desarrollo de estas tareas se utilizó el software MAXQDA, que permitió organizar los datos recolectados para realizar las tareas de comparación constante, saturación y triangulación de fuentes de datos.

\section{Resultados}

Los resultados del estudio se presentan a partir de las preguntas de investigación, y en ese sentido se han organizado en tres secciones; primero se analizan las creencias de los docentes sobre la inclusión; en segundo lugar se analizan las prácticas inclusivas; y por último se discute la configuración de las creencias y las prácticas de inclusión educativa en el contexto de aprendizaje de la LEI. En cada apartado se incluyen datos cualitativos y cuantitativos que permiten una comprensión más profunda del objeto de estudio.

\subsection{Creencias de los docentes sobre la inclusión educativa}

La categoría creencias docentes sobre la inclusión educativa en el contexto de aprendizaje de la lectoescritura inicial (LEI) se compone de dos subcategorías: naturaleza de las diferencias humanas y respuesta educativa.

Por medio de la triangulación de la información se generó la saturación de los códigos, lo que permitió identificar las tendencias sobre las creencias de los docentes que conformaron la muestra. Respecto a la subcategoría naturaleza de las diferencias humanas, la creencia que se identifica es la capacidad de aprendizaje desde la mirada del déficit. Lo anterior se pone de manifiesto al considerar que, si un estudiante presenta barreras de aprendizaje en LEI, se debe de forma exclusiva a sus características personales:

La actitud de los estudiantes es lo que entorpece el proceso de aprendizaje, lo limita en su desempeño. Esto es lo que normalmente se maneja, que si le va mal es que tiene mala actitud, que si no está bien en la clase es porque tiene dificultades (docente 12_taller, comunicación personal, 5 de diciembre de 2019).

Con relación a la subcategoría creencias sobre la respuesta educativa, los docentes manifiestan que la enseñanza del alumnado con barreras de aprendizaje en LEI es responsabilidad de todos. Lo anterior no es coherente con la realidad, ya que es el personal de apoyo (educador especial, psicólogo u orientador educativo) quien finalmente se encarga de la atención del alumnado que requiere sistemas y dispositivos de apoyo en el contexto del aprendizaje de la lectura y la escritura:

Necesitamos un especialista directo para trabajar con los estudiantes y anotar recomendaciones, para así saber qué podemos hacer nosotros en el interior de la clase. (docente 04_entrevista, comunicación personal, 10 de enero de 2020). 
Los hallazgos cuantitativos concuerdan con los hallazgos del análisis cualitativo. La Tabla 1 contiene las frecuencias y porcentajes del cuestionario. En ella se identifica claramente que existe una perspectiva dilemática en cuanto a las creencias sobre la naturaleza de las diferencias de los estudiantes en el contexto de aprendizaje de la LEI, es decir, aunque se reconoce el derecho a la educación, persiste la concepción de la diversidad desde el déficit o dificultad de aprendizaje del estudiante. Las creencias sobre la respuesta educativa son de perspectiva interaccionista, es decir, se valora positivamente el sistema de respuesta inclusivo señalado por la literatura, a saber: a) la colaboración entre docentes, b) la responsabilidad compartida, c) la organización del aula y d) las estrategias de enseñanza.

Sin embargo, durante las observaciones de clase y en las entrevistas de docentes, se identificó que, a excepción de la docente de primer grado, los profesores regulares delegan la atención en el personal docente de apoyo, el cual a su vez no ha logrado concretar un trabajo colaborativo en la comunidad educativa.

Tabla 1. Creencias de los docentes sobre la naturaleza del aprendizaje del estudiante y la respuesta educativa

\begin{tabular}{|c|c|c|c|c|c|c|}
\hline \multirow[t]{2}{*}{ Creencias } & \multicolumn{2}{|c|}{ Individual } & \multicolumn{2}{|c|}{ Dilemática } & \multicolumn{2}{|c|}{ Interaccionista } \\
\hline & $\%$ & $\mathrm{n}$ & $\%$ & $\mathrm{n}$ & $\%$ & $\mathrm{n}$ \\
\hline \multicolumn{7}{|l|}{ Naturaleza de las Diferencias } \\
\hline Origen de las diferencias individuales & 12 & 3 & 40 & 10 & 48 & 12 \\
\hline Modificación de las diferencias & 16 & 4 & 40 & 10 & 44 & 11 \\
\hline \multicolumn{7}{|l|}{ Respuesta educativa } \\
\hline Colaboración entre docentes & 12 & 3 & 12 & 3 & 76 & 19 \\
\hline Responsabilidad & 20 & 5 & 12 & 3 & 68 & 17 \\
\hline Organización en el aula & 4 & 1 & 12 & 3 & 84 & 21 \\
\hline Estrategias de enseñanza & 4 & 1 & 8 & 2 & 88 & 22 \\
\hline
\end{tabular}

Nota: Resultados del cuestionario Creencias y Prácticas Pedagógicas Inclusivas. Participaron 25 docentes. Elaboración propia a partir de los resultados, 2020

\subsection{Prácticas pedagógicas inclusivas}

Las prácticas pedagógicas promueven la participación y el aprendizaje de todo el alumnado, ya que parten de supuestos e interpretaciones que valoran la diversidad y focalizan su acción en las intervenciones educativas. Luego del análisis de la información cualitativa se determinó el uso de las prácticas inclusivas, en las que aparecieron con mayor frecuencia las estrategias orientadas al manejo efectivo del aula, seguidas de las estrategias de agrupamiento y la enseñanza adicional y, en menor escala, las prácticas de adaptación y la promoción de la participación. En el manejo efectivo del aula se privilegian el estímulo verbal, el uso de instrucciones y la organización del ambiente del aula. Uno de los docentes opina al respecto: "La práctica positiva es, 'motivar al estudiante'" (docente-05_Taller, 12 de enero de 2020).

Con relación a las prácticas de agrupamiento, se utilizó el trabajo en equipo heterogéneo y los desdobles. Como estrategias de enseñanza adicional, se identificaron la tutoría entre pares de estudiantes y el refuerzo pedagógico; este último se desarrolla al final del año escolar, cuando el alumnado que ha presentado necesidades de apoyo se enfrenta a un proceso de reprobación. Las prácticas de promoción de la participación consisten en variar las actividades y generar un proyecto de aula de creación literaria. Además, las estrategias de adaptación consisten en prolongar el tiempo para culminar una tarea, el empleo de material didáctico variado y el acompańamiento del educador especial durante las evaluaciones finales (ver Figura 2). 

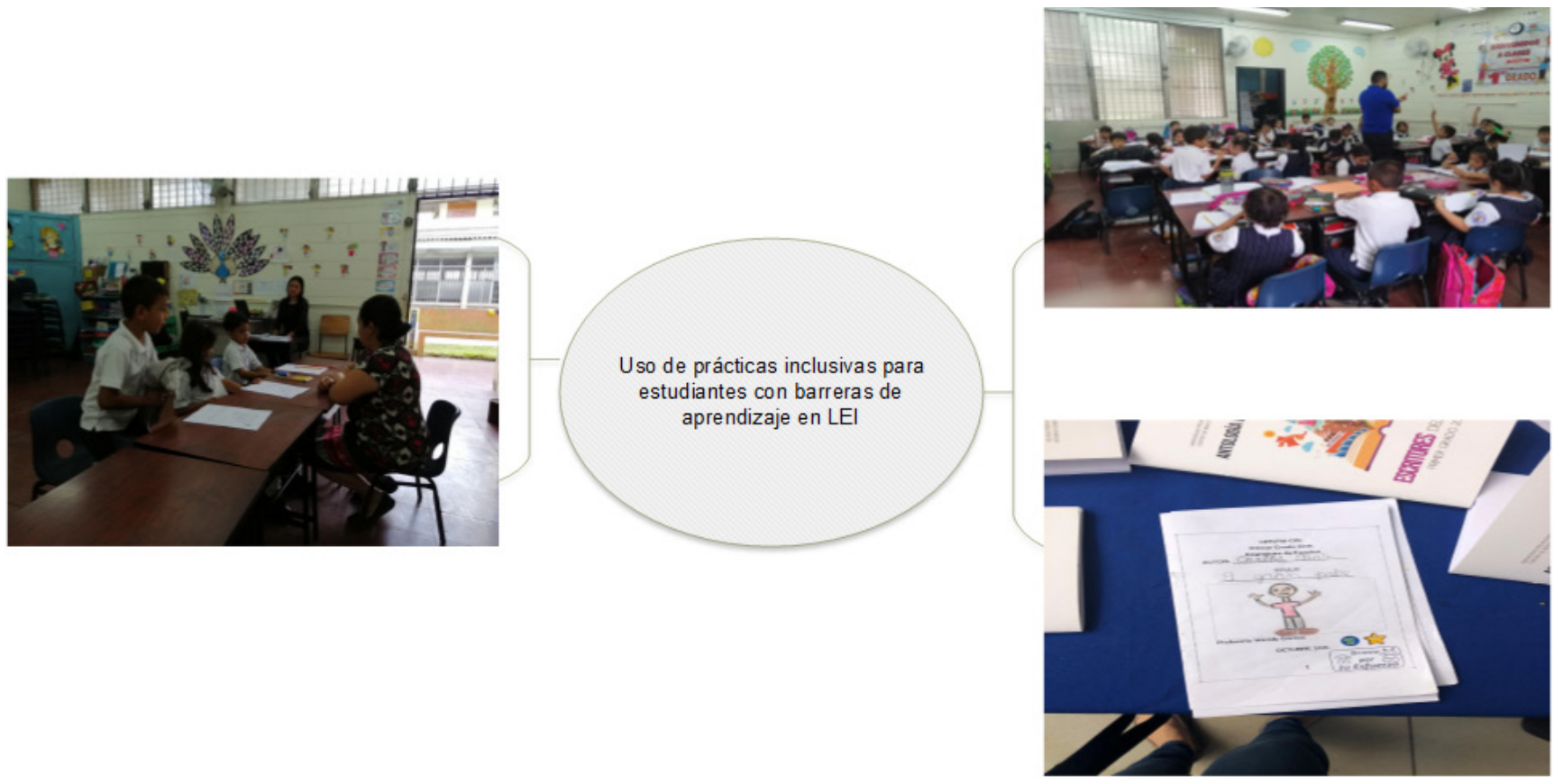

Nota: Elaboración propia, a partir del proceso de observación de aula. Se realizaron seis observaciones.

De igual manera, se analizó la frecuencia de uso de cinco prácticas inclusivas (ver Tabla 2). Los resultados muestran que las estrategias para el manejo efectivo del aula (establecer rutinas, organización del espacio, gestión de la convivencia) son las empleadas con mayor frecuencia por los docentes participantes en el estudio (52\%). En el caso de las estrategias de agrupamiento $(28 \%$, dividir al grupo clase, organizar grupos por capacidades homogéneas y heterogéneas, trabajo en parejas) y la enseñanza adicional (32\%, refuerzo educativo, tutoría, feedback), son poco utilizadas por el profesorado.
En cuanto a las prácticas concernientes a la enseñanza adaptativa (por ejemplo, procesos didácticos y de evaluación diversos, flexibles y significativos), un $56 \%$ de los docentes manifestó emplearlas en alguna medida. De igual forma, el $64 \%$ indicó el uso de las prácticas que apoyan la participación, procurando el uso de materiales y actividades diversas. Es clave destacar en esta dimensión que el $8 \%$ de los profesores manifestó contar con el apoyo del profesor de Educación Especial de forma constante y permanente.

Tabla 2. Porcentaje de uso de las prácticas pedagógicas inclusivas

\begin{tabular}{|c|c|c|c|c|c|c|c|c|}
\hline \multirow{3}{*}{ Práctica } & \multicolumn{8}{|c|}{ Porcentaje } \\
\hline & \multicolumn{2}{|c|}{ Nada } & \multicolumn{2}{|c|}{ Poco } & \multicolumn{2}{|c|}{ Algo } & \multicolumn{2}{|c|}{ Mucho } \\
\hline & $\%$ & $n$ & $\%$ & $n$ & $\%$ & $n$ & $\%$ & $\mathrm{n}$ \\
\hline Manejo efectivo del aula & - & - & - & - & 48 & 12 & 52 & 13 \\
\hline Estrategias de agrupamiento & 4 & 1 & 28 & 7 & 40 & 10 & 28 & 7 \\
\hline Enseñanza adicional & - & - & 32 & 8 & 44 & 11 & 24 & 6 \\
\hline Enseñanza adaptativa & - & - & 20 & 5 & 56 & 14 & 24 & 6 \\
\hline Apoyos a la participación & - & - & 12 & 3 & 64 & 16 & 24 & 6 \\
\hline
\end{tabular}

Nota: Resultados del cuestionario creencias y prácticas pedagógicas inclusivas para la atención de estudiantes en el contexto de la lectura y la escritura. Participaron 25 docentes. Elaboración propia a partir de datos, 2020. 


\subsection{Configuración de creencias y prácticas pedagógicas inclusivas en el contexto de aprendizaje de la LEI}

A partir de la pregunta ¿cómo se configuran las creencias y las prácticas de los docentes para promover la inclusión educativa en el contexto específico del aprendizaje de la LEI?, se realizó un análisis rela- cional entre las categorías, lo que permitió la configuración del fenómeno de estudio. Como muestra la Figura 3, la perspectiva de inclusión educativa identificada en el CIIE fue la perspectiva dilemática, la cual se configura a través de un sistema de creencias y prácticas pedagógicas de los docentes.

Figura 3. Configuración de creencias y prácticas docentes inclusivas en el contexto de aprendizaje de la lectura y la escritura

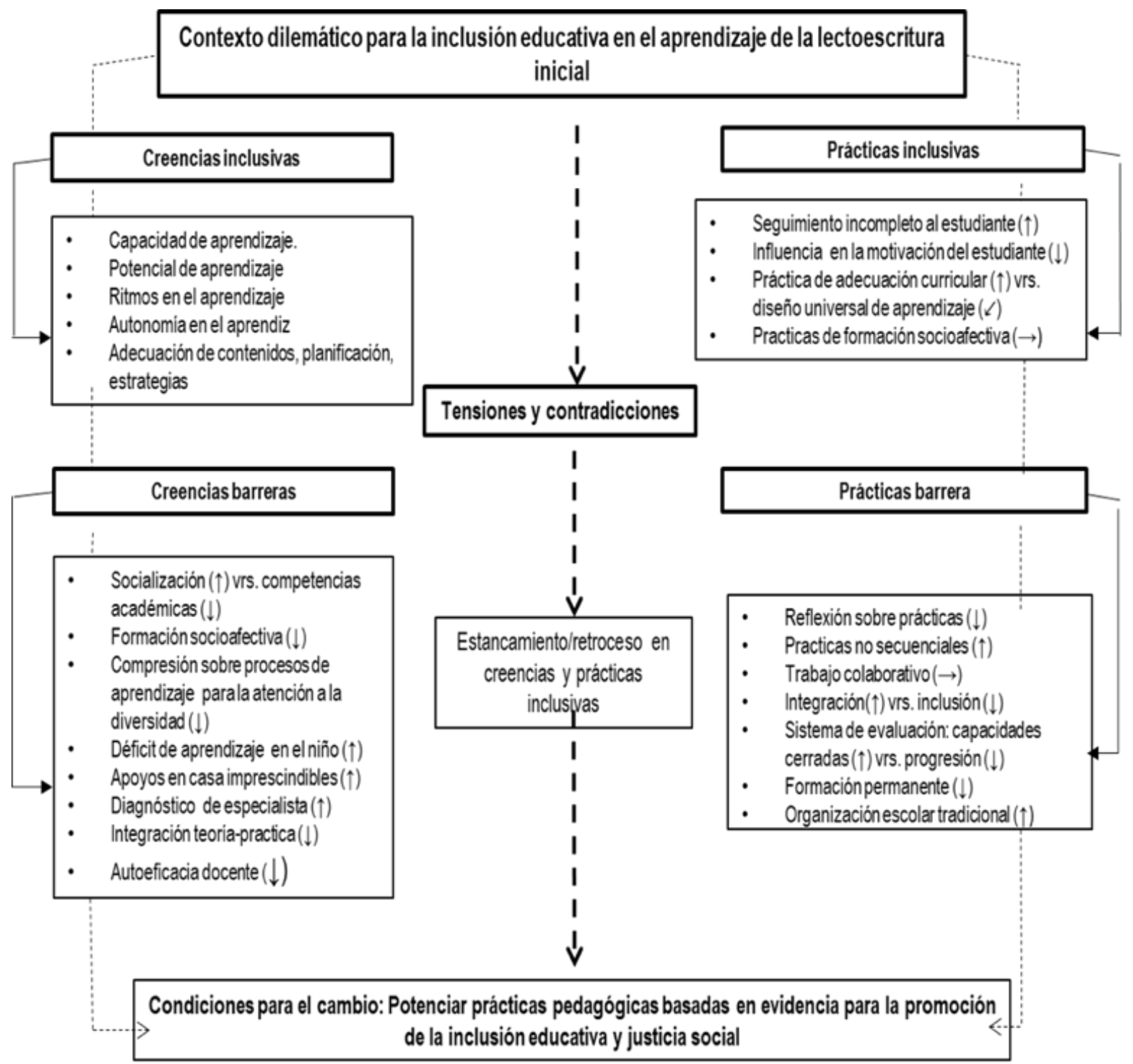

Nota: Elaboración propia a partir de los datos recopilados en el estudio de campo. Se realizaron seis observaciones de aula, un taller investigativo en el que participaron 25 docentes y 10 entrevistas con actores claves que también participaron del taller. 
Con relación a las creencias, se analizaron en dos perspectivas: las creencias inclusivas y las creencias sobre las barreras de aprendizaje y participación. A partir de las entrevistas realizadas a los docentes, las creencias inclusivas identificadas evidencian una concepción dinámica del proceso de aprendizaje, ya que se considera que los estudiantes tienen capacidad y potencial de aprendizaje permanente, por tanto, se requiere identificar los ritmos del aprendiz y respetarlos. Para promover este potencial de aprendizaje, se deben realizar adecuaciones de contenido y, en la planificación, deben integrarse diferentes tipos de estrategias para la atención de los estudiantes.

Respecto a las creencias que se constituyen en barreras para la inclusión, algunos docentes cuestionan que ante las diferencias o dificultades que puedan presentar los estudiantes en su aprendizaje no se deben realizar adecuaciones al currículo, pues todos deben alcanzar los mismos estándares. Sin embargo, otros docentes opinan que este tipo de estudiantes se encuentran en el aula para socializar con sus compañeros de clase, aunque sus rendimientos académicos no sean los esperados. Consideran que esta creencia se convierte en barrera, ya que al final genera una brecha en el logro de competencias académicas. Por otra parte, no se encontró evidencia de que esta mirada de la socialización — que poseen algunos docentes hacia los estudiantes que presentan bajos rendimientos - se articule con un trabajo sistemático para el desarrollo de habilidades socioafectivas vinculadas con aprendizajes de calidad.

Otra creencia barrera que aparece, y que entra en contradicción con las creencias inclusivas sobre el potencial de aprendizaje, es la concepción de que el déficit para aprender se encuentra en el niño, por tanto, son imprescindibles los apoyos en casa y además es central el diagnóstico de un especialista para poder trabajar con el alumnado. En cuanto a la comprensión sobre los procesos de aprendizaje de los estudiantes en LEI, en sus discursos, los docentes expresaron tener escasa formación para atender a los estudiantes que presentan variabilidades en el aprendizaje $y$, aunque consideran que orientarse por la teoría es fundamental, no se ve con claridad que la teoría se conecte con la práctica y que esta relación pueda potenciar los aprendizajes de los estudiantes. Lo anterior influye en el sentido de autoeficacia de los docentes, ya que no tienen claridad respecto a cuáles son las prácticas pedagógicas que mejoran el aprendizaje de los niños y niñas en LEI.

En cuanto a las prácticas inclusivas, gracias a la triangulación de los datos se encontró que el seguimiento de los estudiantes es incompleto, presentándose las siguientes situaciones: a) falta de continuidad en la transición de un grado a otro, en este caso de prebásica al primer grado; y b) durante el año escolar, los procesos quedan inconclusos pues comienzan de forma tardía y le dificultan a los estudiantes mejorar su desempeño. Por otra parte, en las observaciones de aula se identificaron prácticas inclusivas, en las que tiene mayor frecuencia el manejo efectivo del aula (ver Tabla 2), sin embargo, en las entrevistas los docentes las sitúan en la adecuación curricular, lo que corresponde a una perspectiva más individualista y centrada en la integración educativa. Lo anterior evidencia un discurso institucionalizado centrado en el déficit y, por tanto, la estrategia curricular que se implementa en el aula se orienta a adaptar y compensar las diferencias en el aprendizaje, lo que impide avanzar hacia una estrategia curricular basada en un enfoque más interaccionista (como el diseño universal de aprendizaje), la cual no fue identificada durante la investigación.

Respecto a la formación de habilidades socioafectivas, los docentes reportan su desarrollo dentro del aula. Asimismo, en las observaciones de aula se identificó la presencia de material didáctico sobre la temática, sin embargo, en esta investigación no se encontraron evidencias de que estas prácticas se desarrollasen de forma sistemática y orientada a la mejora de los aprendizajes.

Respecto a las prácticas que se constituyen en barreras para el aprendizaje y la participación, el análisis evidenció que la reflexión sobre las prácticas pedagógicas efectivas orientadas a promover la atención a la diversidad es baja; lo anterior puede ser evidencia de que el trabajo colaborativo entre docentes no está 
articulado. Se encontró que el sistema de prácticas está condicionado por el sistema de evaluación que implementa el centro escolar, el cual es regido por las normativas que emite la Secretaría de Educación de Honduras (SEH). Según estas normativas, si bien se promueve la evaluación formativa orientada a valorar la progresión del estudiante, al final prevalece un enfoque sumativo ya que se debe otorgar una calificación final para promover al alumnado al siguiente grado. El énfasis en la evaluación sumativa responde, según los discursos de los docentes, a una concepción tradicional de la organización escolar que viene de las directrices que emite la SEH y por las cuales debe regirse el CIIE. Sobre este punto, a partir del año 2019, la SEH emitió disposiciones para la eliminación de la promoción automática durante el I Ciclo de Educación Básica, por tanto, los niños y niñas pueden reprobar el grado de no alcanzar el $70 \%$ de las evaluaciones que aplican los docentes.

Este tipo de prácticas no inclusivas relacionadas con la evaluación y que son promovidas y reguladas por el sistema educativo tiene implicancias sociales negativas, ya que los niños y niñas, al no contar con un sistema de apoyos pertinente, pueden repetir cursos, provocando dos problemáticas, la sobreedad y el abandono escolar.

\section{Discusión y conclusiones}

Los resultados de esta investigación ponen en evidencian la presencia de creencias orientadas a una perspectiva favorable a la inclusión, respecto a la atención de estudiantes en cuanto a la respuesta educativa. Sin embargo, las creencias sobre la capacidad de aprendizaje del alumnado con dificultades pertenecen a una perspectiva dilemática, este aspecto coincide con los resultados de otros estudios (López et al., 2014; Muñoz et al., 2015). Estos investigadores afirman que, desde esta perspectiva, los docentes debaten cuál es la mejor forma para entregar los apoyos y servicios para los estudiantes y, aunque el profesorado es consciente del derecho a la educación en condiciones de justicia social, ponerlo en práctica resulta bastante complejo (Organización de las Naciones Unidas para la Educación, la Ciencias y la Cultura [UNESCO], 2017), pues persiste la creencia de que hay que centrarse en la capacidad y no en el potencial y resiliencia de cada individuo (García-Carrasco, 2017; García-Carrasco \& Canal-Bedia, 2018). Cuando las creencias se basan en esta perspectiva se piensa que la diversidad en el aprendizaje depende exclusivamente del estudiante $y$, por lo tanto, se generan actuaciones pedagógicas en las que se delega la responsabilidad del trabajo docente en otros profesionales. En contraposición a esta mirada, la firme creencia en que la enseñanza y los dispositivos didácticos pueden contribuir a desarrollar el potencial de aprendizaje generan prácticas pedagógicas inclusivas que contribuyen a eliminar las barreras de aprendizaje y participación del alumnado.

Con relación a las prácticas, se valora que las mismas se adscriben de igual forma a una perspectiva dilemática, situación que coincide con investigaciones previas (Chiner \& Cardona, 2012; Duk \& Murillo, 2016; López et al., 2010; Norwich, 2014, 2019). En los docentes que participaron en este estudio existe un fuerte cuestionamiento sobre la idoneidad de las prácticas empleadas, lo que se suscita por las creencias sobre el potencial de los estudiantes, pero también por la falta de toma de decisiones basadas en evidencia y una cultura pseudocolaborativa (Park et al., 2016). Esto es aún más evidente en las prácticas de delegación, pues se considera que es trabajo de los especialistas y estos, a su vez, realizan su labor de manera aislada (López et al., 2014; McKay \& Manning, 2019). Nuestro estudio ha identificado que las prácticas inclusivas — como la enseñanza adicional, el agrupamiento flexible, la enseńanza adaptativa desde el diseño universal de aprendizaje — son poco utilizadas, esto se debe a la falta de programas de formación permanente y al trabajo colaborativo, aspectos esenciales de un centro educativo inclusivo.

Los hallazgos preliminares sobre cómo se configuran las creencias y prácticas docentes para la inclusión educativa en el contexto de la LEI aportan evidencias sobre la existencia de la perspectiva dilemática, en la respuesta educativa, en donde coexisten una perspectiva individualista y una perspectiva interaccionista. Por tanto, estudiar las creencias ha permitido descu- 
brir la existencia de creencias y prácticas pedagógicas que están en contradicción, ya que en unos momentos se orientan a la integración y otras a la inclusión. Es importante destacar que "los maestros con una disposición más favorable hacia la inclusión utilizan prácticas más inclusivas que aquellos con actitudes menos positivas" (Chiner \& Cardona, 2012, p. 3). En este sentido, encontramos que en el centro predomina una mirada híbrida acerca de la diversidad de los alumnos, pues, aunque se reconoce que las oportunidades educativas son un derecho de todo el alumnado, las prácticas y la cultura generan barreras para los niños con diversidad en el aprendizaje de la LEI.

En síntesis, y atendiendo a las cuestiones de la investigación, se presentan las siguientes conclusiones:

1. Las creencias del profesorado participante sobre los estudiantes con dificultades en el contexto del aprendizaje de la LEI son de naturaleza dilemática. Sin embargo, las concepciones sobre la respuesta educativa se acercan a la perspectiva interaccionista. Esto tiene implicaciones importantes para la atención de los estudiantes, pues la creencia nuclear pone en duda el potencial de aprendizaje y, por lo tanto, como se ha evidenciado en este grupo de profesores, algunas prácticas inclusivas se usan con poca frecuencia y en esfuerzos aislados. Como resultado, los estudiantes con dificultades en el aprendizaje viven un proceso educativo caracterizado por prácticas educativas poco eficaces, que derivan en situaciones de fracaso escolar y estigmas a largo plazo.

2. Respecto a las prácticas pedagógicas inclusivas, el análisis cualitativo y cuantitativo reveló que el manejo efectivo del aula es la práctica por excelencia, y en menor grado se encuentran la enseńanza adaptativa, la enseńanza adicional, el agrupamiento y los apoyos a la participación. No obstante, se pudo constatar que las prácticas carecen de sistematicidad y de un trabajo cooperativo sólido y persistente entre el profesor regular y el profesorado de apoyo, lo que ocasiona una fragmentación en los servicios y dispositivos de apoyo que requiere todo el alumnado y, sobre todo, aquellos con diversidades individuales en el aprendizaje de la LEI.

3. El análisis de la configuración dilemática de las creencias y prácticas inclusivas ha permitido identificar un conjunto de polaridades en las que se mueven los docentes participantes de este estudio, y que dan cuenta de la complejidad que implica trabajar desde una perspectiva inclusiva en educación. Entre las polaridades identificadas aparecen las siguientes:

- Socialización versus formación de competencias académicas

- Integración de teoría versus práctica

- Socialización versus formación socioafectiva

- Adecuación curricular versus diseño universal de aprendizaje

- Evaluación formativa versus evaluación sumativa

- Integración versus inclusión

Estas polaridades provocan tensiones y contradicciones que, al no ser reflexionadas y cuestionadas con base en la evidencia, pueden promover estancamiento o retroceso desde la perspectiva de la inclusión educativa como proceso continuo para promover aprendizajes de calidad desde el centro escolar.

4. En cuanto a las implicaciones, los hallazgos preliminares evidencian que un camino para promover el cambio individual y colectivo es el de una perspectiva del desarrollo profesional docente basado en evidencia, sobre prácticas pedagógicas probadas en investigación y que estén relacionadas con aprendizajes de calidad.

5. En lo relativo a las limitaciones, esta investigación se realizó con un diseño exploratorio de caso único y la selección intencional de los participantes, por tanto, los hallazgos, si bien son valiosos para comprender las creencias y prácticas docentes en un contexto específico, no pueden generalizarse a 
otros contextos, debido a ello, es importante que en futuras investigaciones se amplíe la muestra y la selección sea aleatoria.

\section{Agradecimientos}

Las autoras agradecen a la Red para la Lectoescritura Inicial de Centroamérica y el Caribe (RedLEI) por el financiamiento otorgado para realizar esta investigación a través de la Modalidad de Beca 3, para el desarrollo de investigación y la publicación de artículo académico, y asimismo agradecen la asesoría técnica brindada durante todo el proceso de investigación y publicación.

\section{Referencias bibliográficas}

Booth, T., \& Ainscow, M. (2015). Guía para la Educación Inclusiva. Desarrollando el aprendizaje y la participación en los centros escolares. Madrid: OEI y FUHEM. http://r.issu.edu.do/l.php?l=76M7l

Bravo, L. (2010). Prácticas inclusivas en el aula: validación de un instrumento para conocer la perspectiva del alumnado de primaria y secundaria. Actualidades Investigativas en Educación, 10(3), 18-23. https://doi.org/10.15517/AIE.V10I3.10137

Cardona, M. C. (2003). Inclusión y cambios en el aula vía adaptaciones instructivas. Revista de Investigación Educativa, 21(2), 465-487. http://r.issu.edu. do/l.php?l=77SER

Cardona, M. C. (2006). Diversidad y Educación Inclusiva. Madrid: Pearson.

Chevalier, J. (2009). SAS2: Guí para la Investigación Colaborativa y la Movilización Social. México, D. F.: Centro Internacional de Investigaciones para el Desarrollo (IDRC).

Chiner, E., \& Cardona, M. C. (2012). Inclusive education in Spain: how do skills, resources, and supports affect regular education teachers' perceptions of inclusion? International Journal of Inclusive Education, 17(5), 526-541.

https://doi.org/10.1080/13603116.2012.689864
Chiner, E., \& Cardona, M. C. (2013). Teachers' Use of Inclusive Practices in Spain. The International Journal of Learner Diversity and Identities, 19(1), 2945. https://doi.org/10.18848/2327-0128/CGP/v19i01/48571

Chiner, E., Cardona, M. C., \& Gómez-Puerta, J. M. (2015). Teachers' beliefs about diversity: an analysis from a personal and professional perspective. Journal of New Approaches in Educational Research, 4(1), 1823. https://doi.org/10.7821/naer.2015.1.113

Duk, C., \& Murillo, F. J. (2011). Aulas, escuelas y sistemas educativos inclusivos: la necesidad de una mirada sistémica. Revista Latinoamericana de Educación Inclusiva, 5(2), 11-12. https://bit.ly/32DU2uD

Duk, C., \& Murillo, F. J. (2016). La Inclusión como Dilema. Revista Latinoamericana de Educación Inclusiva, 10(1), 11-14.

https://doi.org/10.4067/S0718-73782016000100001

Durán, D., \& Climent, G. G. (2017). La formación del profesorado para la educación inclusiva: Un proceso de desarrollo profesional y de mejora de los centros para atender la diversidad. Revista Latinoamericana de Educación Inclusiva, 5(2), 153-170. https://bit.ly/2TgDKne

Flores-Barrera, V. J., García-Cedillo, I., \& Romero-Contreras, S. (2017). Inclusive practices in teacher training in Mexico. Liberabit: Revista Peruana de Psicología, 23(1), 39-56.

https://doi.org/10.24265/liberabit.2017.v23n1.03

Florian, L., \& Linklater, H. (2010). Preparing teachers for inclusive education: using inclusive pedagogy to enhance teaching and learning for all. Cambridge Journal of Education, 40(4), 369-386. http://dx.doi.org/10.1080/0305764X.2010.526588

García-Carrasco, J. (2017). Plasticidad, vulnerabilidad e inclusión: tres ejes para la teoría de la educación. Edetania: Estudios y Propuestas Socio-Educativas, (50), 21-46. https://bit.ly/3argwS6

García-Carrasco, J., \& Canal-Bedia, R. (2018). Así somos los humanos: plásticos, vulnerables y resilientes. Salamanca: FahrenHouse. 
Graham, L. J. (2016): Reconceptualising inclusion as participation: Neoliberal buck-passing or strategic by-passing? Discourse: Studies in the Cultural Politics of Education, 37(4), 563-581. https://doi.org/10.1080/01596306.2015.1073021

Hamre, B., \& Oyler, C. (2004). Preparación de maestros para aulas inclusivas: aprendizaje de un grupo de investigación colaborativa. Revista de formación docente, 55(2), 154-163.

https://doi.org/10.1177/0022487103261588

Hart, S., Dixon, A., Drummond, M. J., \& McIntyre, D. (2004). Learning without limits. Londres: Open University Press.

Hoppey, D., Black, W. R., \& Mickelson, A. M. (2018). The Evolution of Inclusive Practice in Two Elementary Schools: Reforming Teacher Purpose, Instructional Capacity, and Data-Informed Practice. International Journal of Educational Reform, 27(1), 2245. https://doi.org/10.1177/105678791802700102

Leko, M. M., Kulkarni, S., Lin, M. C., \& Smith, S. A. (2015). Delving Deeper into the Construct of Preservice Teacher Beliefs About Reading Instruction for Students With Disabilities. Teacher Education and Special Education: The Journal of the Teacher Education Division of the Council for Exceptional Children, 38(3), 186-206.

https://doi.org/10.1177/0888406414557677

López, M., Echeita, G., \& Martín, E. (2009). Concepciones sobre el proceso de inclusión educativa de alumnos con discapacidad intelectual en la educación secundaria obligatoria. Cultura y Educación, 21(4), 485-496. https://bit.ly/2PwL0dt

López, M., Echeita, G., \& Martín, E. (2010). Dilemas en los procesos de inclusión: explorando instrumentos para una comprensión de las concepciones educativas del profesorado. Revista Latinoamericana de Educación Inclusiva, 4(2), 155-176.

https://bit.ly/2wTUskQ

López, V., González, P., Manghi, D., Ascorra, P., Oyanedel, J. C., Redón, S., \& Salgado, M. (2018). Políticas de inclusión educativa en Chile: Tres nu- dos críticos. Education Policy Analysis Archives, 26(3), 157. https://doi.org/10.14507/epaa.26.3088

López, V., Julio, C., Pérez, M., Morales, M., \& Rojas, C. (2014). Barreras culturales para la inclusión: políticas y prácticas de integración en Chile. Revista de Educación, (363), 256-281.

https://doi.org/10-4438/1988-592Xt-RE-2012-363-190

Martínez-Pineda, M. C., \& Soler-Martín, C. (2015). Formación y acción pedagógicas de los maestros. Vínculos entre educación y justicia social. Folios, 26(42), 17-27. https://doi.org/10.17227/01234870.42folios17.27

McKay, L., \& Manning, H. (2019). Do I Belong in the Profession? The Cost of Fitting In as a Preservice Teacher With a Passion for Social Justice. Journal of Teacher Education, 70(4), 360-371.

https://doi.org/10.1177/0022487118811326

Montané, A. (2015). Justicia Social y Educación. Revista de Educación Social (20), 92-113.

https://bit.ly/2VxbNdx

Muñoz-Villa, M. L., López-Cruz, M., \& Assaél, J. (2015). Concepciones docentes para responder a la diversidad: ¿Barreras o recursos para la inclusión educativa? Psicoperspectivas. Individuo y Sociedad, 14(3). https://doi.org/10.5027/psicoperspectivas-Vol14-Issue3-fulltext-646

Norwich, B. (2014). Recognising value tensions that underlie problems in inclusive education. Cambridge Journal of Education, 44(4), 495-510.

https://doi.org/10.1080/0305764X.2014.963027

Norwich, B. (2019). From the Warnock Report (1978) to an Education Framework Commission: A Novel Contemporary Approach to Educational Policy Making for Pupils with Special Educational Needs/Disabilities. Frontiers in Education, 4(4), 495510. https://doi.org/10.3389/feduc.2019.00072

Olson, A. J. \& Roberts, C. A. (2018). Perspectivas de los formadores de docentes: preparación de docentes en servicio para proporcionar acceso al plan de estudios general. Remediación y Educación Especial, 39(6), 365-376.

https://doi.org/10.1177/0741932517738567 
Organización de las Naciones Unidas, para la Educación, la Ciencias y la Cultura (UNESCO). (2017). Guía para asegurar la inclusión y la equidad en la educación. https://bit.ly/2VA0odb

Park, M. H., Dimitrov, D. M., Das, A., \& Gichuru, M. (2016). The teacher efficacy for inclusive practices (TEIP) scale: dimensionality and factor structure. Journal of Research in Special Educational Needs, 16(1), 2-12.

https://doi.org/10.1111/1471-3802.12047

Paz, C. L. (2014). Competencias docentes para la atención a la diversidad: Investigación acción en la Universidad Pedagógica Nacional Francisco Morazán de Honduras (Tesis doctoral). Universidad de Alicante, España. https://bit.ly/2IeM9Tf

Paz, C. L., \& Cardona, M. C. (2018). Percepciones de los docentes en pre-servicio relativas a la inclusión y la atención a la diversidad. Paradigma: Revista de Investigación Educativa, 23(36), 95-110.

https://doi.org/10.5377/paradigma.v23i36.6489

Peebles J., \& Mendaglio S. (2014). Preparación de maestros para aulas inclusivas: introducción del enfoque de experiencia directa individual. Learning Landscapes, 7(2), 245-257.

https://doi.org/10.36510/learnland.v7i2.663

Pegalajar, M. del C., \& Colmenero, M. de J. (2017). Actitudes y formación docente hacia la inclusión en Educación Secundaria Obligatoria. Revista Electróni- ca de Investigación Educativa, 19(1), 84-97. http://redie.uabc.mx/redie/article/view/765

Pugach, M. C., Blanton, L. P., Mickelson, A. M., \& Boveda, M. (2020). Teoría del plan de estudios: la perspectiva que falta en la formación del profesorado para la inclusión. Formación del profesorado y educación especial, 43(1), 85-103.

https://doi.org/10.1177/0888406419883665

Salvador, C. (2013). Guía de buenas prácticas en educación inclusiva. Madrid: Save the Children.

Secretaría de Educación. (2014). Reglamento Educación Inclusiva para personas con discapacidad, necesidades educativas especiales y talentos excepcionales. Honduras. http://r.issu.edu.do/l.php?l=75iYk

Sharma, U. (2018). Preparación para enseñar en aulas inclusivas. Oxford Research Encyclopedia of Education.

https://doi.org/10.1093/acrefore/9780190264093.013.113

Simón, C., Echeita, G., \& Sandoval, M. (2018). Incorporating students' voices in the 'Lesson Study' as a teacher-training and improvement strategy for inclusion. Culture and Education, 30(1), 205-225. https://doi.org/10.1080/11356405.2017.1416741

Vaillant, D., \& Marcelo, C. (2015): El ABC y D de la formación docente. Madrid: Narcea Ediciones.

\section{CÓMO CITAR:}

Paz-Delgado, C., \& Barahona, E. (2020). Creencias y prácticas docentes sobre la inclusión educativa en el contexto de aprendizaje de la lectoescritura inicial. Revista Caribeña de Investigación Educativa (RECIE), 4(2), 112-126. https://doi.org/10.32541/ recie.2020.v4i2.pp112-126 